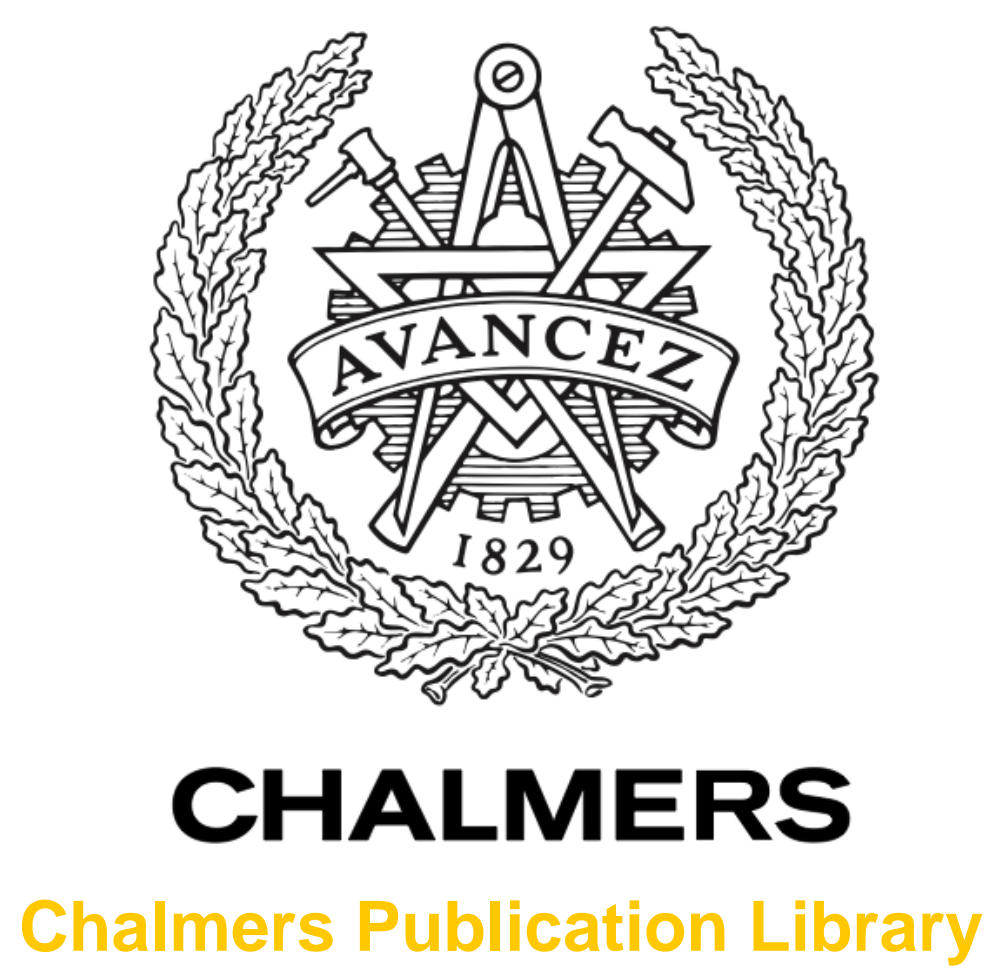

Two Novel Upper Bounds on the Sum Rate of MIMO ZF Receivers

This document has been downloaded from Chalmers Publication Library $(\mathrm{CPL})$. It is the author's version of a work that was accepted for publication in:

54th Annual IEEE Global Telecommunications Conference (GLOBECOM), Houston, DEC 0509, 2011 (ISSN: 1930-529X)

Citation for the published paper:

Matthaiou, M. ; Zhong, C. ; Ratnarajah, T. (2011) "Two Novel Upper Bounds on the Sum Rate of MIMO ZF Receivers". 54th Annual IEEE Global Telecommunications Conference (GLOBECOM), Houston, DEC 05-09, 2011

Downloaded from: http://publications.lib.chalmers.se/publication/156530

Notice: Changes introduced as a result of publishing processes such as copy-editing and formatting may not be reflected in this document. For a definitive version of this work, please refer to the published source. Please note that access to the published version might require a subscription. 


\title{
Two Novel Upper Bounds on the Sum Rate of MIMO ZF Receivers
}

\author{
Michail Matthaiou*, Caijun Zhong ${ }^{\dagger}$, and Tharm Ratnarajah ${ }^{\dagger}$ \\ *Department of Signals and Systems, Chalmers University of Technology, Gothenburg, Sweden \\ ${ }^{\dagger}$ Institute of Electronics, Communications and Information Technology (ECIT), Queen's University Belfast, U.K. \\ E-mail: michail.matthaiou@chalmers.se \{c.zhong, t.ratnarajah\}@ecit.qub.ac.uk
}

\begin{abstract}
In this paper, we introduce two novel upper bounds on the achievable sum rate of multiple-input multiple-output (MIMO) systems with Zero-Forcing (ZF) receivers. The presented bounds are given in tractable closed-form and apply for different fading models, like uncorrelated/doubly correlated Rayleigh fading and Ricean fading. In addition, the first bound establishes an interesting relationship between the sum rate and the first negative moment of the unordered eigenvalue of the instantaneous correlation matrix. Based on our analytical expressions, we are able to explore the impact of the model parameters, such as number of antennas, spatial correlation and Ricean- $K$ factor, on the sum rate of MIMO $\mathrm{ZF}$ receivers.
\end{abstract}

\section{INTRODUCTION}

The capacity of MIMO systems operating with optimal nonlinear receivers, which minimize the error probability, has been exhaustively studied in the corresponding literature [1]-[5]. The main disadvantage of such schemes, however, is their high complexity, especially for large number of antennas, which makes their employment prohibitive in practical applications. For this reason, we will hereafter consider linear ZF receivers, which yield lower complexity compared to other receivers like successive interference cancellation or minimum meansquared error (MMSE) [6], [7]. Surprisingly, the performance of MIMO ZF receivers has not been thoroughly investigated due to the difficulty in statistically characterizing the inverse of the instantaneous correlation matrix.

In the context of MIMO ZF receivers, we first note the work of [7], which derived a closed-form expression for the post-processing Signal-to-Noise ratio (SNR) of the ZF receiver operating in semi-correlated Rayleigh fading, with correlation only on the side with the minimum number of antennas. Later, [8] extended the analysis to the doubly correlated Rayleigh fading channels. However, analytical results were derived only for dual $2 \times 2$ MIMO configurations. The case of uncorrelated and semi-correlated Ricean fading, were respectively addressed in [9] and [10]. Both studies, however, rely on a classical statistical result that approximates a non-central Wishart distribution with a central Wishart distribution, whose accuracy deteriorates at high Ricean $K$-factors. In the context of MIMO MMSE receivers, we recall [11], in which the authors formulated a generic analytical framework for statistically characterizing the sum rate. To the best of our knowledge, a similar analysis for MIMO ZF receivers seems to be missing from the literature. Very recently, [12] compared the high-SNR performance of MIMO ZF and MMSE receivers.
For this reason, using some recent results from random matrix theory, we herein present two novel bounding techniques for the achievable sum rate of MIMO ZF receivers. The derived bounds apply for arbitrary number of antennas and remain tight across the entire SNR range. More importantly, our analysis is generic since it applies for several fading models of interest, namely independent and identically distributed (i.i.d.) and doubly correlated Rayleigh fading along with uncorrelated Ricean fading. We note that the cases of doubly correlated Rayleigh and uncorrelated Ricean fading induce some significant difficulties in the statistical description of the post-processing SNR, and therefore have been rarely examined so far. Despite these inherent difficulties, all the presented analytical expressions can be rather easily evaluated and therefore allow for fast and efficient computation.

Through the first upper bound, we provide a new relationship between the sum rate and the first negative moment of the unordered eigenvalue of the instantaneous correlation matrix. The proposed bounds reveal the impact of the model parameters on the achievable sum rate. For instance, the effects of the number of antennas, SNR, spatial correlation and Ricean $K$-factor are assessed in detail. In fact, in the high-SNR regime the effects of transmit correlation, receive correlation and deterministic LoS component are effectively decoupled. We also provide the link between the presented results for ZF receivers and those reported for optimal/MMSE receivers.

Notation: We use upper and lower case boldface to denote matrices and vectors. The $n \times n$ identity matrix reads as $\mathbf{I}_{n}$ while a $n \times m$ full of zeros matrix as $\mathbf{0}_{n \times m}$. The $(i, j)$ th minor of a matrix is given by $\mathbf{A}_{i j}$, while $\mathbf{A}_{i}$ is $\mathbf{A}$ with the $i$-th column removed. The expectation is given by $\mathcal{E}[\cdot]$ while the matrix determinant and trace by $\operatorname{det}(\cdot)$ and $\operatorname{tr}(\cdot)$. The symbols $(\cdot)^{\dagger}$ and $(\cdot)^{H}$ represent the pseudo-inverse and Hermitian transpose of a matrix, while $\otimes$ is the Kronecker product. The symbol $\sim \mathcal{C N}(\mathbf{M}, \boldsymbol{\Sigma})$ represents a complex Gaussian matrix with mean $\mathbf{M}$ and covariance $\boldsymbol{\Sigma}$. The notation $\Gamma(\cdot)$ expresses the Gamma function, $\operatorname{Ei}(x)=-\int_{-x}^{\infty} \frac{e^{-t}}{t} d t$ the exponential integral function [13, Eq. (8.211.1)], and $\psi(x)$ denotes the digamma function [13, Eq. (8.360.1)].

\section{MIMO Signal Model AND ZF RECEPTION}

We consider a typical point-to-point MIMO system equipped with $N_{r}$ receive antennas and $N_{t}$ transmit antennas with $N_{r} \geq N_{t}$, which is characterized by the fading matrix 
$\mathbf{H} \in \mathbb{C}^{N_{r} \times N_{t}}$. Note that in the following, the instantaneous MIMO correlation matrix $\mathbf{W} \triangleq \mathbf{H}^{H} \mathbf{H}$, will be extensively used. Assuming no channel state information at the transmitter, the available power, $P$, is uniformly distributed to all data streams. The discrete-time input-output relationship reads as

$$
\mathbf{y}=\sqrt{\frac{P}{N_{t}}} \mathbf{H} \mathbf{s}+\mathbf{n}
$$

where $\mathbf{y} \in \mathbb{C}^{N_{r} \times 1}$ is the received signal vector, $\mathbf{s} \in \mathbb{C}^{N_{t} \times 1}$ is the vector containing the transmitted symbols which are drawn from a unit-power constellation; the complex noise term is Gaussian distributed with zero-mean and covariance $\mathcal{E}\left[\mathbf{n n}^{H}\right]=N_{0} \mathbf{I}_{N_{r}}$, where $N_{0}$ is the noise power. We now apply the concept of ZF reception on (1) to obtain the ZF filter matrix according to $\mathbf{G}=\left(P / N_{t}\right)^{-1 / 2} \mathbf{H}^{\dagger}$ [6]. Using the simple detection scheme of [7], we consider that each component of $\hat{\mathbf{s}}$ is independently decoded and the received signal after detection becomes

$$
\hat{\mathbf{s}}=\mathbf{G} \mathbf{y}=\mathbf{s}+\left(P / N_{t}\right)^{-1 / 2} \mathbf{H}^{\dagger} \mathbf{n}
$$

with the instantaneous received SNR at the $m$-th ZF output being equal to [6], [7]

$$
\gamma_{m} \triangleq \frac{\rho}{N_{t}\left[\left(\mathbf{H}^{H} \mathbf{H}\right)^{-1}\right]_{m m}}, m=1, \ldots, N_{t}
$$

where $\rho=P / N_{0}$ is the average SNR and $[\cdot]_{m m}$ returns the $m$-th diagonal element of a matrix. The achievable sum rate is essentially the sum of throughputs contributed from all subchannels, or

$$
R \triangleq \sum_{m=1}^{N_{t}} \mathcal{E}\left[\log _{2}\left(1+\gamma_{m}\right)\right]
$$

where the expectation is taken over all channel realizations of $\mathbf{H}$ and the channel is assumed to be ergodic. A sum rate analysis of MIMO ZF receivers requires precise knowledge of the statistics of $\gamma_{m}$ which however are available only for i.i.d Rayleigh or semi-correlated Rayleigh fading channels [7].

\section{GENERIC SUM RATE BOUNDS}

We now derive two novel generic bounds on the sum rate of MIMO ZF receivers that apply for a finite number of antennas and for arbitrary SNRs. We begin with the following theorem which returns an upper bound on the sum rate:

Theorem 1: The achievable sum rate of MIMO ZF receivers is upper bounded by $R \leq R_{u, 1}$ with

$$
\begin{aligned}
R_{u, 1} & =N_{t} \log _{2}\left(\mathcal{E}\left[\lambda^{-1}\right]+\frac{\rho}{N_{t}}\right)+\frac{N_{t}}{\ln 2} \mathcal{E}\left[\ln \left(\operatorname{det}\left(\mathbf{H}^{H} \mathbf{H}\right)\right)\right] \\
& -\frac{1}{\ln 2} \sum_{m=1}^{N_{t}} \mathcal{E}\left[\ln \left(\operatorname{det}\left(\mathbf{H}_{m}^{H} \mathbf{H}_{m}\right)\right)\right]
\end{aligned}
$$

where $\lambda$ is an unordered eigenvalue of $\mathbf{W}$.
Proof: Starting from (4), we can obtain

$$
\begin{aligned}
R & =\sum_{m=1}^{N_{t}} \mathcal{E}\left[\log _{2}\left(1+\frac{\rho}{N_{t}} \frac{1}{\left[\mathbf{W}^{-1}\right]_{m m}}\right)\right] \\
& =\sum_{m=1}^{N_{t}} \mathcal{E}\left[\log _{2}\left(\left[\mathbf{W}^{-1}\right]_{m m}+\frac{\rho}{N_{t}}\right)-\log _{2}\left(\left[\mathbf{W}^{-1}\right]_{m m}\right)\right] \\
& =\sum_{m=1}^{N_{t}} \mathcal{E}\left[\log _{2}\left(\left[\mathbf{W}^{-1}\right]_{m m}+\frac{\rho}{N_{t}}\right)\right] \\
& -\sum_{m=1}^{N_{t}} \mathcal{E}\left[\log _{2}\left(\left[\mathbf{W}^{-1}\right]_{m m}\right)\right]
\end{aligned}
$$

The first term in (6) can be successively upper bounded by

$$
\begin{aligned}
1_{\text {term }} & \leq N_{t} \mathcal{E}\left[\log _{2}\left(\frac{1}{N_{t}} \sum_{m=1}^{N_{t}}\left(\left[\mathbf{W}^{-1}\right]_{m m}+\frac{\rho}{N_{t}}\right)\right)\right] \\
& =N_{t} \mathcal{E}\left[\log _{2}\left(\frac{1}{N_{t}} \operatorname{tr}\left(\mathbf{W}^{-1}\right)+\frac{\rho}{N_{t}}\right)\right] \\
& \leq N_{t} \log _{2}\left(\frac{1}{N_{t}} \mathcal{E}\left[\operatorname{tr}\left(\mathbf{W}^{-1}\right)\right]+\frac{\rho}{N_{t}}\right)
\end{aligned}
$$

where from (6) to (7) we have used the inequality of arithmetic and geometric means, while (9) is a result of Jensen's inequality. Combining (6) with (9) and with the aid of the following key matrix property [7], [11],

$$
\left[\mathbf{W}^{-1}\right]_{m m}=\frac{\operatorname{det}\left(\mathbf{W}_{m m}\right)}{\operatorname{det}(\mathbf{W})}=\frac{\operatorname{det}\left(\mathbf{H}_{m}^{H} \mathbf{H}_{m}\right)}{\operatorname{det}\left(\mathbf{H}^{H} \mathbf{H}\right)}
$$

we conclude the proof.

Clearly, the evaluation of (5) requires the existence of the first negative moment of the unordered eigenvalue and as such the following lemma will be particularly useful:

Lemma 1 ([14]): For a continuous random variable $X$ with probability density function $f_{X}(x)$, its first negative moment does not exist if it has a positive mass at $X=0$, i.e. $f(0)>0$.

Theorem 2: The achievable sum rate of MIMO ZF receivers is upper bounded by $R \leq R_{u, 2}$ with

$$
\begin{aligned}
R_{u, 2} & =\sum_{m=1}^{N_{t}} \log _{2}\left(\mathcal{E}\left[\operatorname{det}\left(\mathbf{H}_{m}^{H} \mathbf{H}_{m}\right)\right]+\frac{\rho}{N_{t}} \mathcal{E}\left[\operatorname{det}\left(\mathbf{H}^{H} \mathbf{H}\right)\right]\right) \\
& -\frac{1}{\ln 2} \sum_{m=1}^{N_{t}} \mathcal{E}\left[\ln \left(\operatorname{det}\left(\mathbf{H}_{m}^{H} \mathbf{H}_{m}\right)\right)\right] .
\end{aligned}
$$

Proof: The derivation of the second upper bound is based on the subsequent methodology

$$
\begin{aligned}
R=\sum_{m=1}^{N_{t}} \mathcal{E}\left[\log _{2}\left(1+\frac{\rho}{N_{t}} \frac{1}{\left[\mathbf{W}^{-1}\right]_{m m}}\right)\right] \\
=\sum_{m=1}^{N_{t}} \mathcal{E}\left[\log _{2}\left(\operatorname{det}\left(\mathbf{W}_{m m}\right)+\frac{\rho}{N_{t}} \operatorname{det}(\mathbf{W})\right)\right. \\
\left.-\log _{2}\left(\operatorname{det}\left(\mathbf{W}_{m m}\right)\right)\right]
\end{aligned}
$$

where from (12) to (13) we have used (10). Applying Jensen's inequality on (13), we can easily obtain (11). 


\section{SUM RATE BOUNDS IN FADING CHANNELS}

\section{A. Uncorrelated Rayleigh fading}

The uncorrelated Rayleigh model represents a rich scattering environment with no direct path and large antenna spacings. In this case, the fading matrix can be modeled as

$$
\mathbf{H}=\mathbf{H}_{w}
$$

where the entries of $\mathbf{H}_{w}$ entries are i.i.d. complex zero-mean, unit-variance random variables.

Proposition 1: For i.i.d. Rayleigh fading, the sum rate of MIMO ZF receivers is upper bounded by

$$
\begin{aligned}
R_{u, 1} & =N_{t} \log _{2}\left(\frac{1}{N_{r}-N_{t}}+\frac{\rho}{N_{t}}\right)+\frac{N_{t}}{\ln 2} \psi\left(N_{r}-N_{t}+1\right) \\
R_{u, 2} & =N_{t} \log _{2}\left(\frac{N_{r} !}{\left(N_{r}-N_{t}+1\right) !}+\frac{\rho}{N_{t}} \frac{N_{r} !}{\left(N_{r}-N_{t}\right) !}\right) \\
& -\frac{N_{t}}{\ln 2} \sum_{k=1}^{N_{t}-1} \psi\left(N_{r}+1-k\right) .
\end{aligned}
$$

Proof: The proof starts by invoking the following results for an $N_{r} \times N_{t}$ (with $N_{r} \geq N_{t}$ ) central Wishart matrix

$$
\begin{aligned}
\mathcal{E}\left[\operatorname{det}\left(\mathbf{H}_{w}^{\dagger} \mathbf{H}_{w}\right)\right] & =\frac{N_{r} !}{\left(N_{r}-N_{t}\right) !} \\
\mathcal{E}\left[\ln \left(\operatorname{det}\left(\mathbf{H}_{w}^{\dagger} \mathbf{H}_{w}\right)\right)\right] & =\sum_{k=0}^{N_{t}-1} \psi\left(N_{r}-k\right)
\end{aligned}
$$

which are respectively obtained from [2, Eq. (A.7.1)] and [2, Eq. (A.8.1)]. The unordered eigenvalue of $\mathbf{H}_{w}^{\dagger} \mathbf{H}_{w}$ has the following distribution [1]

$$
f_{\lambda}(x)=\frac{1}{N_{t}} \sum_{k=0}^{N_{t}-1} \frac{k !\left(L_{k}^{N_{r}-N_{t}}(x)\right)^{2}}{\left(N_{r}-N_{t}+k\right) !} e^{-x} x^{N_{r}-N_{t}}
$$

where $L_{k}^{r}(\cdot)$ denotes the generalized Laguerre polynomial of order $k$ [13, Eq. (8.970.1)]. We note that the above distribution has a zero mass at $x=0$ only if $N_{r}-N_{t}>0$, since $L_{k}^{0}(0)=1$; hence, the first negative moment of $\lambda$ is [15, Lemma 6]

$$
\mathcal{E}\left[\frac{1}{\lambda}\right]=\frac{1}{N_{r}-N_{t}}, \text { for } N_{r} \geq N_{t}+1
$$

Substituting (17), (18) and (20) into (5), (11), we can obtain (15), (16) after noting that

$$
\mathbf{H}_{m} \sim \mathcal{C N}\left(\mathbf{0}_{N_{r} \times N_{t}-1}, \mathbf{I}_{N_{r}} \otimes \mathbf{I}_{N_{t}-1}\right)
$$

and some simple simplifications.

Corollary 1: For i.i.d. Rayleigh fading, $R_{u, 1}$ becomes exact at high SNRs and equal to

$$
\begin{aligned}
R_{u, 1}^{\infty} & =N_{t} \log _{2}\left(\frac{\rho}{N_{t}}\right)+\frac{N_{t}}{\ln 2} \psi\left(N_{r}-N_{t}+1\right) \\
& \leq N_{t} \log _{2}\left(\frac{\rho}{N_{t}}\right)+\frac{1}{\ln 2} \sum_{k=0}^{N_{t}-1} \psi\left(N_{r}-k\right) .
\end{aligned}
$$

Proof: Taking $\rho$ large in (15) and simplifying.
We note that (23) corresponds to the high-SNR ergodic capacity of an i.i.d. Rayleigh fading MIMO system with optimal receivers under $N_{r} \geq N_{t}$ [2, Eq. (9)], [4, Eq. (12)]. In addition, (22), (23) validate the well-known feature of MIMO systems, that is the asymptotically (in terms of SNR) linear sum rate (or capacity) scaling with the minimum number of antennas [1]-[5]. Note also that (22) is strongly related with [11, Proposition 2] which was derived in the context of MIMO MMSE receivers; at high-SNRs, both receivers behave equivalently in terms of sum rate [6]. In Fig. 1, the simulated achievable sum rate along with the proposed analytical bounds of (15) and (16) are plotted against the average SNR, $\rho$. We consider different MIMO configurations by keeping $N_{t}=3$ and increasing $N_{r}$.

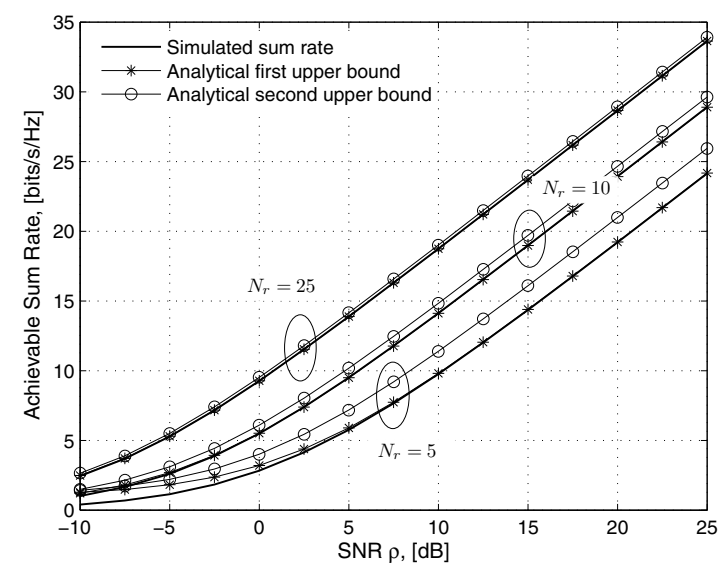

Fig. 1. Simulated sum rate, and upper bounds against the $\operatorname{SNR} \rho\left(N_{t}=3\right)$.

The graph indicates that adding more receive antennas significantly stabilizes the MIMO link by improving the receive diversity and reducing the noise enhancement effect. Clearly, all bounds remain sufficiently tight across the entire SNR range, while $R_{u, 1}$ becomes exact even at moderate SNRs. Regarding $R_{u, 2}$, its tightness improves with an increasing $N_{r}$. In fact, by defining the high-SNR bound offset, as $\Delta R_{u} \triangleq R_{u, 2}^{\infty}-R_{u, 1}^{\infty}=N_{t} \log _{2}\left(\mathcal{E}\left[\operatorname{det}\left(\mathbf{H}^{H} \mathbf{H}\right)\right]\right)-$ $N_{t} \mathcal{E}\left[\log _{2}\left(\operatorname{det}\left(\mathbf{H}^{H} \mathbf{H}\right)\right)\right]$, we can see that in the limit of highSNR and large number of receive antennas, $R_{u, 2}$ becomes exact, due to the law of large numbers $\lim _{N_{r} \rightarrow \infty} \mathbf{H}_{w}^{\dagger} \mathbf{H}_{w} / N_{r} \rightarrow$ $\mathbf{I}_{N_{t}}$.

\section{B. Doubly correlated Rayleigh fading}

This scenario is suitable when spatial correlation occurs on both sides of the MIMO link and as such, we get

$$
\mathbf{H}=\mathbf{R}_{\mathrm{R}}^{1 / 2} \mathbf{H}_{w} \mathbf{R}_{\mathrm{T}}^{1 / 2}
$$

where $\mathbf{R}_{\mathrm{T}} \in \mathbb{C}^{N_{t} \times N_{t}}, \mathbf{R}_{\mathrm{R}} \in \mathbb{C}^{N_{r} \times N_{r}}$ denote the Hermitian, positive definite transmit and receive correlation matrices. In general, this scenario of doubly correlated Rayleigh fading is not amenable to tractable manipulations due to the random term $\operatorname{det}\left(\mathbf{H}_{w}^{H} \mathbf{R}_{\mathrm{R}} \mathbf{H}_{w}\right)$. However, based on some recent advances in random matrix theory, we now present the following result: 
Lemma 2: For semi-correlated Rayleigh fading with correlation on the side with the maximum number of antennas, the first moment of the generalized variance is equal to

$$
\mathcal{E}\left[\operatorname{det}\left(\mathbf{H}_{w}^{H} \mathbf{R}_{\mathrm{R}} \mathbf{H}_{w}\right)\right]=\frac{N_{t} ! \operatorname{det}(\mathbf{X})}{\prod_{i<j}^{N_{r}}\left(\gamma_{j}-\gamma_{i}\right)}
$$

where $\gamma_{i}, i=1, \ldots, N_{r}$ are the real, positive eigenvalues of $\mathbf{R}_{\mathrm{R}}$, while $\mathbf{X}$ is a $N_{r} \times N_{r}$ matrix with entries

$$
\{\mathbf{X}\}_{i, j}=\left\{\begin{array}{cc}
\gamma_{i}^{j-1}, & j=1, \ldots, N_{r}-N_{t} \\
\gamma_{i}^{j}, & j=N_{r}-N_{t}+1, \ldots, N_{r} .
\end{array}\right.
$$

Proof: A detailed proof is given in Appendix I.

We emphasize the fact that the result in (25) is rather tractable and, at the same time, applies for arbitrarydimensional fading matrices $\mathbf{H}$.

Proposition 2: For doubly correlated Rayleigh fading, the sum rate of MIMO ZF receivers is upper bounded by

$$
\begin{aligned}
R_{u, 2} & =\sum_{m=1}^{N_{t}} \log _{2}\left(\frac{\left(N_{t}-1\right) !\left(\operatorname{det}(\tilde{\mathbf{X}})+\frac{\rho}{\sigma_{m}} \operatorname{det}(\mathbf{X})\right)}{\prod_{i<j}^{N_{r}}\left(\gamma_{j}-\gamma_{i}\right)}\right) \\
& -\frac{N_{t}}{\ln 2}\left(\sum_{k=1}^{N_{t}-1} \psi(k)+\frac{\sum_{k=N_{r}-N_{t}+2}^{N_{r}} \operatorname{det}\left(\mathbf{Y}_{k}\right)}{\prod_{i<j}^{N_{r}}\left(\gamma_{j}-\gamma_{i}\right)}\right)
\end{aligned}
$$

where $\sigma_{m}$ is the $m$-th diagonal entry of $\mathbf{R}_{\mathrm{T}}^{-1}$. The matrix $\widetilde{\mathbf{X}}$ is directly related to $\mathbf{X}$ as

$$
\{\widetilde{\mathbf{X}}\}_{i, j}=\left\{\begin{array}{cc}
\gamma_{i}^{j-1}, & j=1, \ldots, N_{r}-N_{t}+1 \\
\gamma_{i}^{j}, & j=N_{r}-N_{t}+2, \ldots, N_{r}
\end{array}\right.
$$

while $\mathbf{Y}_{k}$ is an $N_{r} \times N_{r}$ matrix with entries

$$
\left\{\mathbf{Y}_{k}\right\}_{i, j}=\left\{\begin{array}{cc}
\gamma_{i}^{j-1}, & j \neq k \\
\gamma_{i}^{j-1} \ln \gamma_{i}, & j=k
\end{array}\right.
$$

Proof: A detailed proof is relegated in [16].

Regarding the upper bound $R_{u, 1}$, its evaluation requires statistical knowledge of the unordered eigenvalue, which, however, is an extremely challenging problem. For this reason, we now focus on the still practical case of correlation on the side with the maximum number of antennas (i.e. $\mathbf{R}_{\mathrm{T}}=\mathbf{I}_{N_{t}}$ ):

Corollary 2: For semi-correlated Rayleigh fading with correlation on the side with the maximum number of antennas, the upper bound $R_{u, 1}$ does not exist.

Proof: The proof starts by considering the marginal density of $\lambda$, which for the case of correlation on the receive side reads as [17, Eq. (14)]

$$
\begin{aligned}
f_{\lambda}(x) & =\frac{1}{N_{t} \prod_{i<j}^{N_{t}}\left(\gamma_{j}-\gamma_{i}\right)} \\
& \times \sum_{\ell=1}^{N_{r}} \sum_{k=N_{r}-N_{t}+1}^{N_{r}} \frac{x^{N_{t}-N_{r}+k-\ell} e^{-x / \gamma_{\ell}} \gamma_{\ell}^{N_{r}-N_{t}-1}}{\Gamma\left(N_{t}-N_{r}+k\right)} D_{\ell k}
\end{aligned}
$$

Setting $k=N_{r}-N_{t}+1$, we can see that $f_{\lambda}(0) \neq 0$.
We note that in (26) the effects of transmit and receive correlation are decoupled although the overall impact of receive correlation is not straightforwardly inferred due to the Vandermonde determinants. For this reason, we consider the high-SNR regime via the following corollary:

Corollary 3: For doubly correlated Rayleigh fading, the sum rate at high SNRs becomes

$$
\begin{aligned}
R^{\infty} & =N_{t} \log _{2}\left(\frac{\rho}{N_{t}}\right)+\frac{N_{t}}{\ln 2} \psi\left(N_{t}\right) \\
& -\sum_{m=1}^{N_{t}} \log _{2}\left(\sigma_{m}\right)+\frac{N_{t}}{\ln 2} \frac{\operatorname{det}\left(\mathbf{Y}_{N_{r}-N_{t}+1}\right)}{\prod_{i<j}^{N_{r}\left(\gamma_{j}-\gamma_{i}\right)}} \\
& \leq N_{t} \log _{2}\left(\frac{\rho}{N_{t}}\right)+\frac{1}{\ln 2} \sum_{k=1}^{N_{t}} \psi(k) \\
& +\log _{2} \operatorname{det}\left(\mathbf{R}_{\mathrm{T}}\right)+\frac{k=\sum_{r}-N_{t}+1}{\ln 2 \prod_{i<j}^{N_{r}}\left(\gamma_{j}-\gamma_{i}\right)} .
\end{aligned}
$$

Proof: The proof follows by taking $\rho$ large in (4), using Theorem 2 and simplifying.

Note that (29) corresponds to the average MIMO mutual information of a doubly correlated Rayleigh fading MIMO system with optimal receivers under $N_{r} \geq N_{t}$. To the best of our knowledge the expression in (29) is new and does not require the MIMO matrix to be square, as in [5, Eq. (88)]. What is more, the expression in (28) is in agreement with an associated result on MIMO MMSE receivers [11, Proposition 6]. In Fig. 2, the analytical high-SNR approximations for ZF and optimal receivers are compared, based on (28) and (29).

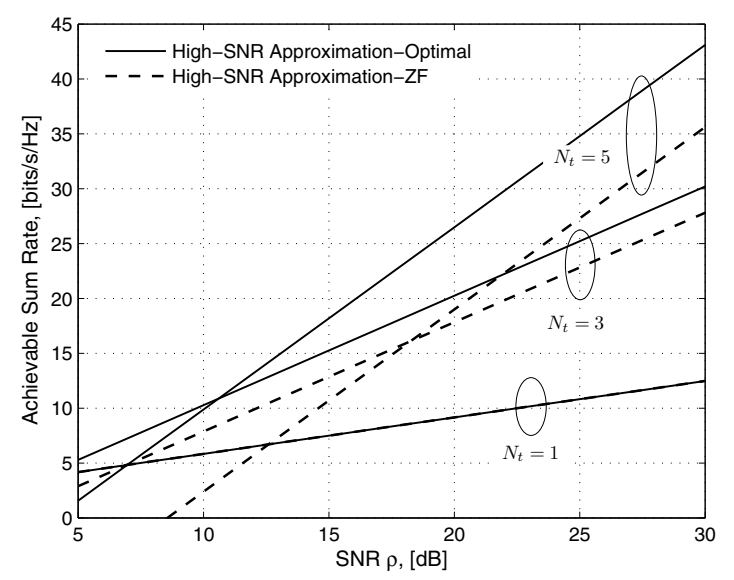

Fig. 2. Analytical high-SNR approximations for ZF and optimal receivers against the $\operatorname{SNR} \rho\left(N_{r}=7, \rho_{t}=0.3, \rho_{r}=0.8\right)$.

The entries of the receive correlation matrix (and likewise of $\mathbf{R}_{\mathrm{T}}$ ) are modeled as $\left\{\mathbf{R}_{\mathrm{R}}\right\}_{i, j}=\rho_{r}^{|i-j|}$ with $\rho_{r} \in[0,1)$ being the receive correlation coefficient. We can clearly observe that as $N_{t}$ increases, the offset between the two expressions increases which demonstrates the increasingly poor interference cancellation capabilities of ZF receivers. 


\section{Uncorrelated Ricean fading}

This model is suitable when there is a direct LoS or specular path between the transmitter and receiver. In order to avoid the introduction of extra cumbersome notations, we hereafter focus on the case of rank-1 deterministic component [11], [18], [19]. Then, the fading matrix $\mathbf{H}$ becomes

$$
\mathbf{H}=\sqrt{\frac{K}{K+1}} \mathbf{H}_{\mathrm{L}}+\sqrt{\frac{1}{K+1}} \mathbf{H}_{w}
$$

where $K$ stands for the Ricean $K$-factor. We first analytically derive the first negative moment of an arbitrary eigenvalue:

Theorem 3: For uncorrelated Ricean fading with a rank$1 \mathrm{LoS}$ component, the first negative moment of an arbitrary eigenvalue, $\lambda$, of $\mathbf{H}^{H} \mathbf{H}$ is (for $N_{r} \geq N_{t}+1$ )

$$
\begin{aligned}
& \mathcal{E}\left[\frac{1}{\lambda}\right]=\frac{e^{-\Delta}(K+1)}{N_{t}\left(\left(N_{r}-N_{t}\right) !^{N_{t}}\right)} \sum_{\ell=1}^{N_{t}} \frac{1}{\Delta^{N_{t}-1} \prod_{i=1}^{N_{t}-1} \Gamma(i)} \\
& \times\left(\sum_{k=1}^{N_{t}-1} \frac{\Gamma\left(N_{r}-N_{t}+\ell+k-2\right)}{(K+1)^{(k-1)}\left(N_{r}-N_{t}+1\right)_{k-1}} \mathcal{A}_{k \ell}\right. \\
& +\Gamma\left(N_{r}-N_{t}+\ell-1\right) \\
& \left.\times{ }_{1} F_{1}\left(N_{r}-N_{t}+\ell-1 ; N_{r}-N_{t}+1 ; \Delta\right) \mathcal{A}_{N_{t} \ell}\right)
\end{aligned}
$$

where $\Delta=K N_{t} N_{r}$, while ${ }_{p} F_{q}(\cdot)$ is the generalized hypergeometric function [13, Eq. (9.14.1)]. The term $\mathcal{A}_{k \ell}$ denotes the $(k, \ell)$-th cofactor of the $N_{t} \times N_{t}$ matrix $\mathcal{A}$ whose entries are

$$
\{\mathcal{A}\}_{i, j}=\left\{\begin{array}{ccrl}
\frac{\left(N_{r}-N_{t}+i+j-2\right) !}{\left(N_{r}-N_{t}+1\right)_{i}-1}, & & 1 \leq i \leq N_{t}-1 \\
\frac{{ }_{1} F_{1}\left(N_{r}-N_{t}+j ; N_{r}-N_{t}+1 ; \Delta\right)}{\left(\left(N_{r}-N_{t}+j-1\right) !\right)^{-1}}, & & i=N_{t}
\end{array}\right.
$$

while $(\alpha)_{n}=\Gamma(\alpha+n) / \Gamma(\alpha)$ is the Pochhammer symbol [13].

Proof: A detailed proof is given in [16].

Proposition 3: For uncorrelated Ricean fading with a rank$1 \mathrm{LoS}$ component, the sum rate of MIMO ZF receivers is upper bounded by

$$
\begin{aligned}
R_{u, 1} & =N_{t} \log _{2}\left(\mathcal{G}(\lambda)+\frac{\rho}{N_{t}}\right)+\frac{N_{t}}{\ln 2}\left(\psi\left(N_{r}-N_{t}+1\right)\right. \\
& \left.+g\left(N_{r}, \Delta\right)-g\left(N_{r}, \Delta_{1}\right)-\ln (K+1)\right) \\
R_{u, 2}= & N_{t} \log _{2}\left(\frac{N_{r} !}{\left(N_{r}-N_{t}+1\right) !} \frac{1+K\left(N_{t}-1\right)}{(K+1)^{N_{t}-1}}\right. \\
+ & \left.\frac{\rho}{N_{t}} \frac{N_{r} !}{\left(N_{r}-N_{t}\right) !} \frac{1+K N_{t}}{(K+1)^{N_{t}}}\right) \\
- & \frac{N_{t}}{\ln 2}\left(g\left(N_{r}, \Delta_{1}\right)-\left(N_{t}-1\right) \ln (K+1)\right. \\
& \left.\quad+\sum_{k=1}^{N_{t}-2} \psi\left(N_{r}-k\right)\right)
\end{aligned}
$$

where $\mathcal{G}(\lambda)=\mathcal{E}\left[\frac{1}{\lambda}\right], \Delta_{1}=K\left(N_{t}-1\right) N_{r}$, while the auxiliary function $g(n, x)$ is defined according to

$$
\begin{aligned}
& g(n, x) \triangleq \ln (x)-\operatorname{Ei}(-x) \\
& +\sum_{k=1}^{n-1}\left(-\frac{1}{x}\right)^{k}\left(e^{-x}(k-1) !-\frac{(n-1) !}{k(n-k-1) !}\right) .
\end{aligned}
$$

Proof: A detailed proof is given in Appendix II.

Since it is difficult to obtain insights into the implications of the Ricean $K$-factor from (32) and (33) we now focus on the high-SNR regime:

Corollary 4: For uncorrelated Ricean fading with a rank-1 LoS component, $R_{u, 1}$ becomes exact at high SNRs and equal to

$$
\begin{aligned}
& R_{u, 1}^{\infty}=N_{t} \log _{2}\left(\frac{\rho}{N_{t}}\right)+\frac{N_{t}}{\ln 2} \psi\left(N_{r}-N_{t}+1\right) \\
& +\frac{N_{t}}{\ln 2}\left(g\left(N_{r}, \Delta\right)-g\left(N_{r}, \Delta_{1}\right)-\ln (K+1)\right) \\
& \leq N_{t} \log _{2}\left(\frac{\rho}{N_{t}}\right)+\frac{1}{\ln 2} \sum_{k=1}^{N_{t}-1} \psi\left(N_{r}-k\right) \\
& +\frac{1}{\ln 2} g\left(N_{r}, \Delta\right)-N_{t} \log _{2}(K+1) .
\end{aligned}
$$

Proof: Taking $\rho$ large in (32) and simplifying.

From (36), we conjecture the deleterious effects of the rank1 deterministic component on the sum rate, since the term $g\left(N_{r}, \Delta\right)-g\left(N_{r}, \Delta_{1}\right)-\ln (K+1)$ was numerically found to be a monotonically decreasing function in $K$. Note that (36) corresponds to the high-SNR ergodic capacity of a rank1 uncorrelated Ricean fading MIMO system with optimal receivers under $N_{r} \geq N_{t}$ [19, Eq. (38)]. In Fig. 3, the effects of Ricean $K$-factor on the performance of the proposed bounds are investigated.

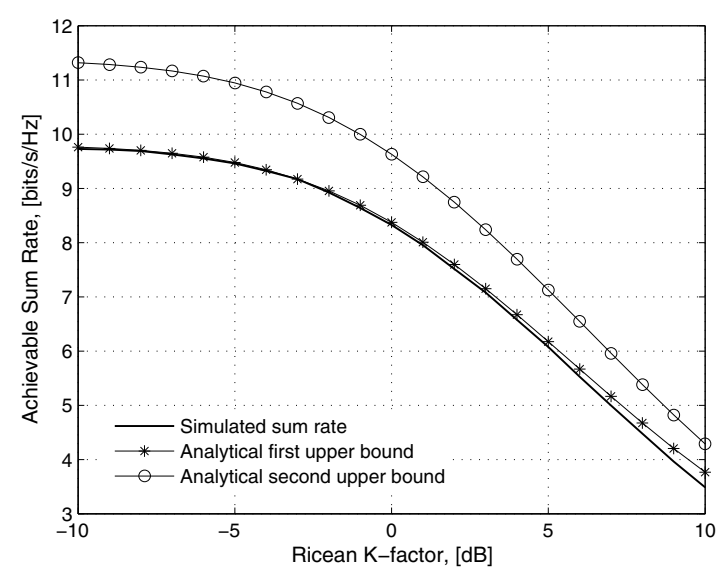

Fig. 3. Simulated sum rate, and upper bounds against the Ricean $K$-factor $\left(N_{r}=5, N_{t}=3, \rho=10 \mathrm{~dB}\right)$.

It can be observed that a higher $K$-factor extensively limits the advantages of MIMO technology for the considered rank1 configurations. This observation is in line with [18], [19]. This can be justified by considering the high-SNR/high $K$ factor regime, which leads to the following insightful result:

$$
R_{u, 1}^{\infty} \stackrel{K \rightarrow \infty}{{ }^{\infty}} N_{t} \log _{2}\left(\frac{\rho}{K N_{t}}\right)+\frac{N_{t}}{\ln 2} \psi\left(N_{r}-N_{t}+1\right)
$$

where we have taken $K$ large in (35) and used the fact that $g\left(N_{r}, \Delta\right) \approx \ln (\Delta)$ for $\Delta \rightarrow \infty$ [19]. We finally note that $R_{u, 1}$ remains notably tight across the entire $K$-factor regime, while $R_{u, 2}$ becomes tighter with an increasing $K$-factor. 


\section{CONCLUSION}

This paper introduced two novel upper bounds on the sum rate of MIMO receivers, that can be applied for arbitrary number of antennas and remain sufficiently tight across the entire SNR range. Both bounds were evaluated for Rayleigh and Ricean fading models and were shown to admit very tractable manipulations. Through the analytical expressions, we gained valuable insights into the implications of the various system and fading parameters on the performance of ZF receivers. An interesting relationship between the sum rate and the first negative moment of the unordered eigenvalue of the instantaneous correlation matrix was also provided.

\section{APPENDIX I \\ ProOF OF THEOREM 2}

The proof is based on a similar methodology as that in [17, Appendix I-E]. More specifically, by simple rearrangement of [17, Eq. (128)] we can get

$$
\mathcal{E}\left[\operatorname{det}\left(\mathbf{H}_{w}^{H} \mathbf{R}_{\mathrm{R}} \mathbf{H}_{w}\right)\right]=\frac{\operatorname{det}(\boldsymbol{\Delta})}{\prod_{i=1}^{N_{t}} \Gamma\left(N_{t}-i+1\right) \prod_{i<j}^{N_{r}}\left(\gamma_{j}-\gamma_{i}\right)}
$$

where $\boldsymbol{\Delta}$ is an $N_{r} \times N_{r}$ matrix with entries

$$
\{\boldsymbol{\Delta}\}_{i, j}=\left\{\begin{array}{c}
\gamma_{i}^{j-1}, \quad j=1, \ldots, N_{r}-N_{t} \\
\gamma_{i}^{N_{r}-N_{t}-1} \int_{0}^{\infty} \lambda^{j-N_{r}+N_{t}} e^{-\lambda / \gamma_{i}} d \lambda, \\
j=N_{r}-N_{t}+1, \ldots, N_{r} .
\end{array}\right.
$$

The second branch of $\Delta$ can be further simplified through [13, Eq. (3.381.4)], to yield

$$
\begin{aligned}
& \{\boldsymbol{\Delta}\}_{i, j} \\
& =\gamma_{i}^{j} \Gamma\left(j-N_{r}+N_{t}+1\right), \quad j=N_{r}-N_{t}+1, \ldots, N_{r} .
\end{aligned}
$$

The proof concludes after factorizing the common terms from $\operatorname{det}(\boldsymbol{\Delta})$ in (37) and simplifying.

\section{APPENDIX II}

\section{PROOF OF PROPOSITION 3}

The proof starts by invoking the following results for a $N_{r} \times$ $N_{t}$ (with $N_{r} \geq N_{t}$ ) noncentral Wishart matrix

$$
\begin{aligned}
\mathcal{E}\left[\operatorname{det}\left(\mathbf{H}^{H} \mathbf{H}\right)\right] & =\frac{N_{r} !}{\left(N_{r}-N_{t}\right) !} \frac{\left(1+K N_{t}\right)}{(K+1)^{N_{t}}} \\
\mathcal{E}\left[\ln \left(\operatorname{det}\left(\mathbf{H}^{H} \mathbf{H}\right)\right)\right] & =g\left(N_{r}, \Delta\right)-N_{t} \ln (K+1) \\
& +\sum_{k=1}^{N_{t}-1} \psi\left(N_{r}-k\right)
\end{aligned}
$$

which are respectively obtained from [19, Theorem 1], [19, Theorem 2]. We note that $g\left(N_{r}, 0\right)=\psi\left(N_{r}\right)$. For Rayleigh fading conditions (i.e. $K=0$ ) (39)-(40) coincide with (17)(18). In order to evaluate the last term in (5), we have

$$
\mathbf{H}_{m} \sim \mathcal{C N}\left(\sqrt{\frac{K}{K+1}} \mathbf{H}_{\mathrm{L}, m}, \frac{1}{K+1} \mathbf{I}_{N_{r}} \otimes \mathbf{I}_{N_{t}-1}\right)
$$

where $\mathbf{H}_{\mathrm{L}, m}$ corresponds to $\mathbf{H}_{\mathrm{L}}$ with the $m$-th column removed. Clearly, we can apply the results of Theorem 3 and
(40) on (5) to obtain (32). Likewise, the upper bound in (33) is again obtained through (41) with the aid of (39)-(40).

\section{ACKNOWLEDGMENTS}

The work of M. Matthaiou has been supported in part by the Swedish Governmental Agency for Innovation Systems (VINNOVA) within the VINN Excellence Center Chase. The work of C. Zhong and T. Ratnarajah was supported by the Future and Emerging Technologies (FET) Programme within the Seventh Framework Programme for Research of the European Commission under FET-Open grant number CROWN-233843 and also by the U.K. Engineering and Physical Sciences Research Council under Grant EP/G026092/1.

\section{REFERENCES}

[1] I. E. Telatar, "Capacity of multi-antenna Gaussian channels," Europ. Trans. Telecommun., vol. 10, no. 6, pp. 585-595, Nov./Dec. 1999.

[2] A. Grant, "Rayleigh fading multi-antenna channels," EURASIP J. Appl. Signal Process., vol. 2002, no. 3, pp. 316-329, Mar. 2002.

[3] H. Shin and J. H. Lee, "Capacity of multi-antenna fading channels: Spatial fading correlation, double scattering, and keyhole," IEEE Trans. Inf. Theory, vol. 49, no. 10, pp. 2636-2647, Oct. 2003.

[4] Ö. Oyman, R. Nabar, H. Bölcskei, and A. Paulraj, "Characterizing the statistical properties of mutual information in MIMO channels," IEEE Trans. Signal Process., vol. 51, no. 11, pp. 2782-2795, Nov. 2003.

[5] M. R. McKay and I. B. Collings, "General capacity bounds for spatially correlated Rician MIMO channels," IEEE Trans. Inf. Theory, vol. 51, no. 9, pp. 3121-3145, Sep. 2005.

[6] A. Paulraj, R. Nabar, and D. A. Gore, Introduction to Space-Time Wireless Communications, Cambridge University Press, May 2003.

[7] D. A. Gore, R. W. Heath, Jr., and A. Paulraj, "Transmit selection in spatial multiplexing systems," IEEE Commun. Lett., vol. 6, no. 11, pp. 491-493, Nov. 2002.

[8] M. Kiessling and J. Speidel, "Analytical performance of MIMO zeroforcing receivers in correlated Rayleigh fading environments," in Proc. IEEE Signal Process. Advances Wireless Commun. (SPAWC), Rome, Italy, June 2003, pp. 383-387.

[9] R. Xu and F. C. M. Lau, "Performance analysis for MIMO systems using zero-forcing detector over fading channels," IEE Proc. Commun., vol. 153, no. 1, pp. 74-80, Feb. 2006.

[10] H. A. Abou Saleh and W. Hamouda, "Performance of zero-forcing detectors over MIMO flat-correlated Ricean fading channels," IET Commun., vol. 3, no. 1, pp. 10-16, Jan. 2009.

[11] M. R. McKay, I. B. Collings, and A. M. Tulino, "Achievable sum rate of MIMO MMSE receivers: A general analytic framework," IEEE Trans. Inf. Theory, vol. 56, no. 1, pp 396-410, Jan. 2010.

[12] J. Yi, M. K. Varanasi, and L. Jian, "Performance analysis of ZF and MMSE equalizers for MIMO systems: An in-depth study of the high SNR regime," IEEE Trans. Inf. Theory, vol. 57, no. 4, pp. 2008-2026, Apr. 2011.

[13] I. S. Gradshteyn and I. M. Ryzhik, Table of Integrals, Series, and Products, Seventh Ed. Academic Press, San Diego, 2007.

[14] W. W. Piegorsch and G. Casella, "The existence of the first negative moment," The American Statistician, vol. 39, no. 1, pp. 60-62, Feb. 1985.

[15] A. Lozano, A. M. Tulino, and S. Verdú, "Multiple-antenna capacity in the low-power regime," IEEE Trans. Inf. Theory, vol. 49, no. 10, pp. 2527-2544, Oct. 2003.

[16] M. Matthaiou, C. Zhong and T. Ratnarajah, "Novel generic bounds on the sum rate of MIMO ZF receivers," in press IEEE Trans. Signal Process., vol. 59, 2011.

[17] S. Jin, M. R. McKay, C. Zhong, and K. -K. Wong, "Ergodic capacity analysis of amplify-and-forward MIMO dual-hop systems," IEEE Trans. Inf. Theory, vol. 56, no. 5, pp. 2204-2224, May 2010.

[18] M. R. McKay and I. B. Collings, "On the capacity of frequency-flat and frequency-selective Rician MIMO channels with single-sided correlation," IEEE Trans. Wireless Commun., vol. 5, no. 8, pp. 2038-2043, Aug. 2005.

[19] S. Jin, X. Gao, and X. You, "On the ergodic capacity of rank-1 Riceanfading MIMO channels," IEEE Trans. Inf. Theory, vol. 53, no. 2, pp. 502-517, Feb. 2007. 